\title{
Application of Brillouin-based distributed optical fibre sensing technology to measure strain development of a slope model
}

\author{
Dayangku Salma Awang Ismail ${ }^{1,2 *}$, Azman Kassim $^{3}$, Hisham Mohamad ${ }^{4}$, Ahmad Safuan A. \\ Rashid $^{3}$, Aliff Ridzuan Bunawan ${ }^{1}$ \\ ${ }^{1}$ Faculty of Civil Engineering, Universiti Teknologi Malaysia, 81310 Johor Bahru, Johor, Malaysia \\ ${ }^{2}$ Faculty of Engineering, Universiti Malaysia Sarawak, 94300 Kota Samarahan, Sarawak, Malaysia \\ ${ }^{3}$ Associate Professor, Faculty of Civil Engineering, Universiti Teknologi Malaysia, 81310 Johor \\ Bahru, Johor, Malaysia \\ ${ }^{4}$ Associate Professor, Civil \& Environmental Engineering Dept., UniversitiTeknologi PETRONAS, \\ 32610 Seri Iskandar, Perak DarulRidzuan, Malaysia
}

\begin{abstract}
For almost two decades, distributed optical fibre sensors are well-known for an alternative to conventional instrumentation in geotechnical engineering applications. However, the technology is yet to be fully implemented due to uncertainties of attachment method or the best way to deploy optical fibre for geo-structure health monitoring. Thus, a project of a $1 \mathrm{~g}$ model of soil slope was intiated and was constructed with three layers of optical fibre that were horizontally embedded in the soil slope mass in order to observe strain development due to a surcharge load. The strain mobilizations were measured by using Brillouin Optical TimeDomain Analysis (BOTDA) sensing system during the incremental loading on the slope crest until a failure feature had been initiated. The aim of study is to evaluate the development of horizontal strains from Brillouinbased optical fibre sensor subjected to soil slope deformation which lead to slope failures. The results showed that the measurands of optical fibre were highly accumulated at the position of $0.3 \mathrm{~m}$ depth from the slope crest. The development of high strain at this position was because of soil-fibre interaction to the overburden imposed load in perpendicular direction of optical fibre placement. Therefore, it can be concluded that the optical fibre strain in the soil-strain field were well-responded to the particle soil movement. In addition, the significant trend of positive strain curves were illustrated when the soil was under compression due to external load from a surcharge load plus self-weight of the soil material.
\end{abstract}

\section{Introduction}

Optical fibre technology has been employed in structural health monitoring industry in more than two decades and seemed has been positively appraised by the users. It is due to

\footnotetext{
*Corresponding author: dayangsalma@gmail.com
} 
robust characteristics to electrical interference compared to other electrical-based instrumentations which have had been utilized past several decades as a sensor to monitor infrastructure decays. The design of optical fibre sensor is to allow changes of glass properties when light is passing through subjected to different light intensity, polarization and spectral content of phase [1]

There were several types of optical fibre sensor used in civil engineering application includes Fibre-Bragg grating sensor, SOFO (long-gauge optical fibre sensor) and the timedomain reflectometry-based sensor (distributed optical fibre sensor). The Fibre Bragg grating sensor was firstly discovered by Hill et al 1978 [2] as a photo-generated gratings in germanosilicate optical fibres. The measurement principles of a Bragg grating is when a Bragg wavelength, $\lambda_{B}$ was shifted proportionally with axial strain or temperature variation due to changes of the sensor segment through a periodic variation of refractive index along the length of the fibre [3] As for SOFO, Leung et al., 2013 has mentioned that the sensor includes two single-mode fibres which located in a tube and attached to the host structure; one is named as measurement fibre (attached to the structure) and the other named as reference fibre positioned in a loose condition. The deformation would take into account when a difference in length was occurred between these two (2) fibres. Finally, the optical time-domain reflectometry system (OTDRs) was designed for telecommunications industry and known as the basic of distributed optical sensing technology.

\subsection{The principle of distributed optical sensing technique}

The working principle of optical time-domain reflectometry is defined when the attenuation of pulse light along a distance of optical fibre was evaluated subjected to a back-scattered signal and was measured with respect to time-scale. The time domain is changed to distance information by multiplying by half the group light speed in the fibre. In the OTDR technique, the Rayleigh scattered light is the basis of attenuation profiles of optical fibre. The detected signal from the scattered light was directly related to time and linear towards the end of fibre. Therefore, the OTDR profiles obtained were very beneficial to signify the location of splices and connectors, optical breaks and generally evaluate the whole fibre [1]. There are three types of scattering-based signal; Rayleigh, Raman and Brillouin scattering. The back-scattered signal was occurred due to changes of refractive index in optical fibre after a light wave travels in an inhomogeneous medium [4]

According to Leung et al 2013[1], the frequency of Rayleigh scattering is almost similar to the probe pulse and so that limits the distance range. Nonetheless, Raman and Brillouin back-scatter signal have illustrated a different wavelength compared to Rayleigh scattering. As shown in Figure 1, the Raman scattered light has a very broad wavelength from $10-13$ $\mathrm{THz}$ and is highly depended on temperature changes but not really sensitive to strain measurand. Meanwhile, Brillouin scattering is measured from the gain (or loss) of signal regards to interaction of two-counter propagating waves; optical pulse and continuous wave (CW). Besides, the uniqueness of Brillouin OTDR system also has able to measure for both strain and temperature due to its narrow frequency shift $(10-13 \mathrm{GHz})$ and natural linewidth which is approximately about $30 \mathrm{MHz}$.

Horiguchi and Tateda in 1989 [5] has introduced a new measurement technique to monitor light wave attenuation in optical fibre which they named it as Brillouin optical time-domain analysis (BOTDA). The concept of BOTDA is based on Stimulated Brillouin Scattering (SBS) in an optical fibre when two light waves; continuous light wave and pump pulse light wave in counter propagated direction are coupled and frequency difference between these two lights named the Brillouin frequency shift between the light waves is then matched to the Brillouin frequency of optical fibre core which dependence of strain and temperature variations [6] This characteristics has enabled a distributed sensing system 
that can provide a complete profile of strain distribution which an advantageous to civil engineering application.

\subsection{The concept of BOTDA}

The concept of BOTDA sensing system is when two counter-propagating light waves in a single-mode optical fibre interchange energy along the fibre due to inhomogeneties of silica glass. The pump pulse wave is launched at one end of optical fibre and the other continuous wave $(\mathrm{CW})$ is propagated in the opposite direction of the optical pulse. Barrias et al 2016 [7] stated that the energy conservation between pump and probe beam (CW) has successively resulted in an amplification of stimulated Brillouin gain at maximum when the difference between two light beams known as Brillouin frequency shift $\left(v_{B}\right)$ is equal to local Brillouin frequency shift, $v_{B O}$. In addition, the $v_{B}$ were varies accordingly to mechanical and thermal states of the fibre. Therefore, the Brillouin scattering wave principle enable the direct measurement of longitudinal strains and temperature variation of optical fibre. [8]

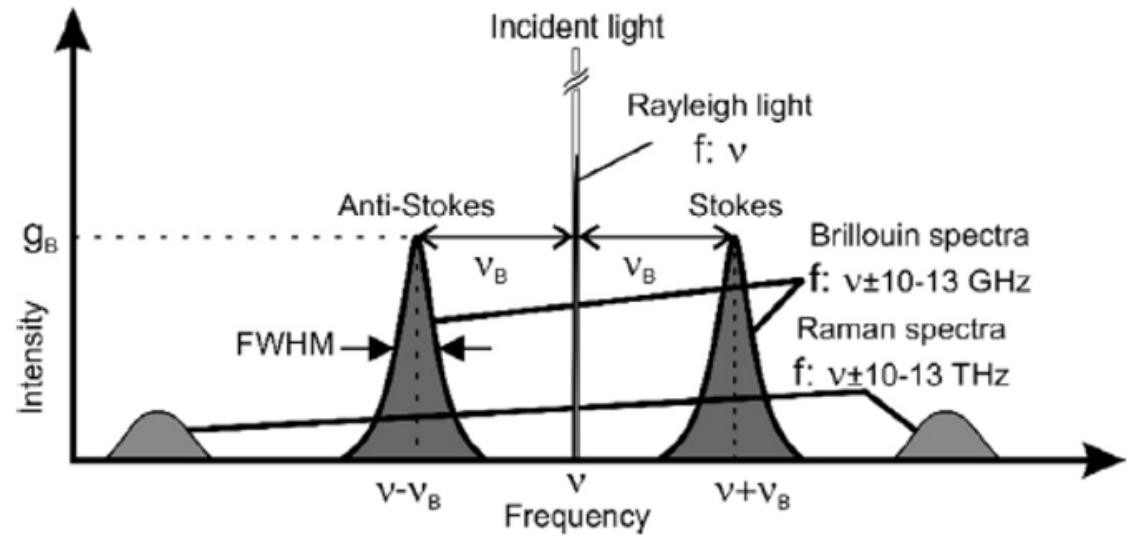

Fig. 1. Brillouin scattering spectrum (FWHM stands for the full width at half maximum. [9] 


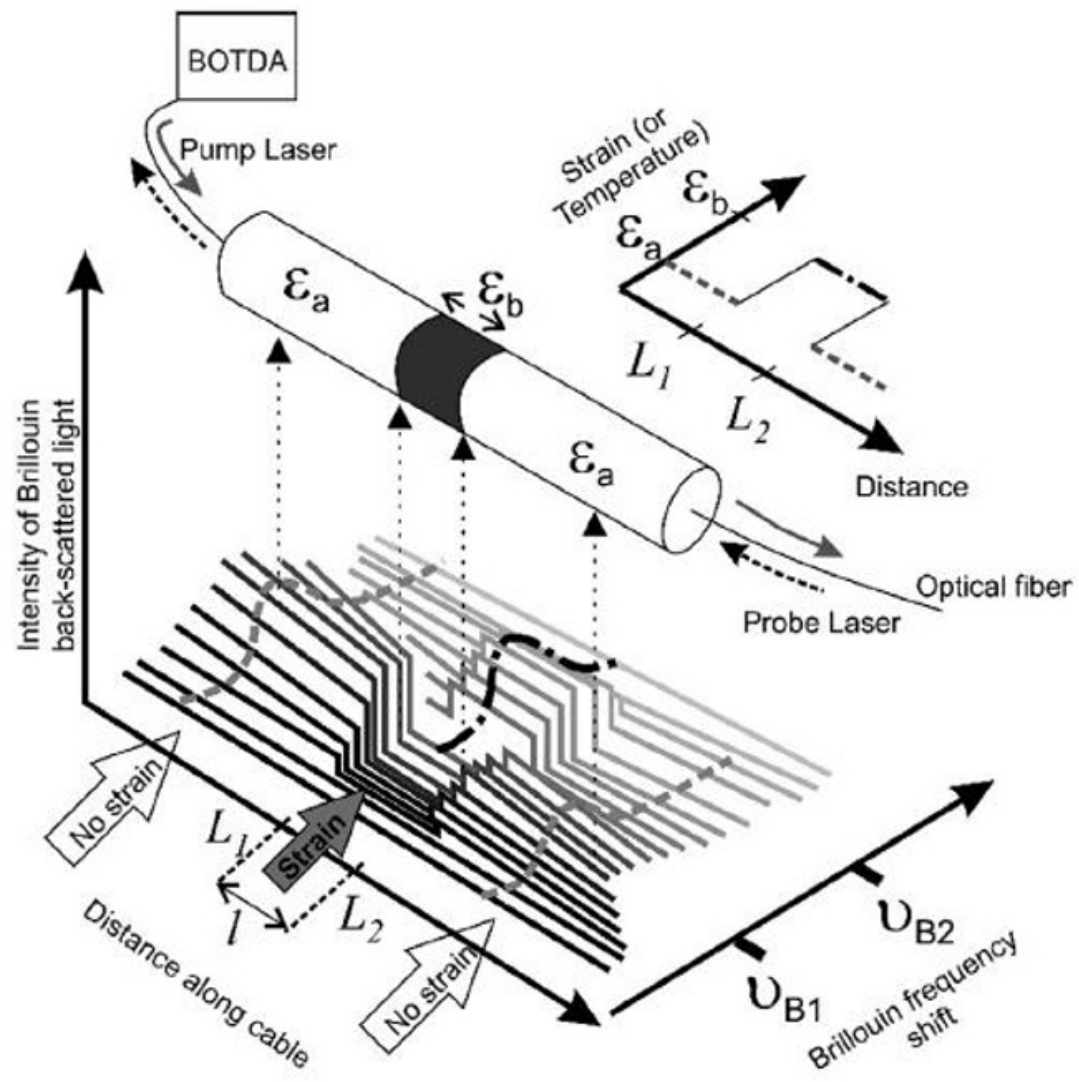

Fig. 2. The stimulated Brillouin gain is maximised when the frequency difference between pump and probe light equals to the local Brillouin frequency shift (BFS). [9]

\section{Material and Methods}

There were five laboratory schemes had been conducted included one pilot test for the doctoral project. The laboratory schemes were involved three major direct measurements; remote positioning tensiometer to measure negative pore-pressure, optical fibre to measure soil strain and digital single-lens reflex (DSLR) camera to capture digital image of soil slope deformation. In this paper, only one model scheme; no rain with 27 degree slope inclination were elaborated and were only focused on the strain distribution of optical fibre within the soil slope mass.

Table 1. Laboratory schemes of the project.

\begin{tabular}{|c|c|c|c|c|}
\hline $\begin{array}{c}\text { Experiment } \\
\text { No. }\end{array}$ & Soil Configuration & $\begin{array}{c}\text { Slope } \\
\left.\text { Angle } \mathbf{(}^{(}\right)\end{array}$ & $\begin{array}{c}\text { Rainfall Intensity } \\
\mathbf{( m / s )}\end{array}$ & Remarks \\
\hline- & $\begin{array}{c}\text { Homogenous slope } \\
\text { (pilot test) }\end{array}$ & 27 & $1.51 \times 10^{-5}$ & 2 hours rain \\
\hline 1a & Homogenous slope & 27 & - & 0 hour rain \\
\hline 1b & Homogenous slope & 27 & $2.46 \times 10^{-5}$ & 1 hour rain \\
\hline 1c & Homogenous slope & 27 & $2.53 \times 10^{-6}$ & 24 hours rain \\
\hline
\end{tabular}




\begin{tabular}{|c|c|c|c|c|}
\hline $2 \mathrm{~b}$ & Homogenous slope & 45 & $2.46 \times 10^{-5}$ & 1 hour rain \\
\hline $2 \mathrm{c}$ & Homogenous slope & 45 & $2.53 \times 10^{-6}$ & 24 hours rain \\
\hline
\end{tabular}

Figure 2 illustrates the three-dimensional mechanism of Brillouin gain spectrum for stimulated Brillouin signal for a BOTDA system. As the BOTDA sensing system has been utterly responsive to the variation of temperature and strain along the cable, this characteristic has made the system performed a distributed measurements. Therefore, the Brillouin shift profile of a medium can be obtained using the BOTDA sensing system as the stimulated Brillouin signal is a function of time and frequency. Then, the measurands (strain/temperature) were computed as a result of the translated frequency shift, $\left(v_{B}\right)$ via the use of quantified calibration coefficient of an optical fibre. The captured profiles (frequency shift difference, $v_{B}$ ) are then can be interpreted as the medium overall deformation or temperature readings as stated in Equation 1[8]

$$
v_{B}=v_{B 0}+C_{\varepsilon} \Delta \varepsilon+C_{T} \Delta T
$$

where ;

$v_{B}=$ Brillouin frequency shift

$v_{B 0}=$ initial Brillouin frequency

$C_{\varepsilon}=$ strain dependent calibration coefficient

$C_{T}=$ temperature dependent calibration coefficient

$\Delta \varepsilon=$ changes of strain at subsequent measurments

$\Delta T=$ changes of temperature at subsequent measurments

The complete distributed strain profile were identified along an optical fibre when the BOTDA system ran through the entire length of cable at specified parameter configurations, i.e spatial resolution and sampling intervals [10] Because of these configurations, the Brillouin frequency shift actually was measured in a discrete manner due to sampling points which set apart at a constant interval of measurement (spatial resolution). The strain readings are calculated by averaging of frequency difference shift over a spatial resolution. According to Omnisens DITEST ${ }^{\mathrm{TM}}$ (2018) [11] for fibre optic distributed temperature and strain sensing technique technical note, the spatial resolution can be defined as the instrument's capability to measure two adjacent locations and directly related to pulse width of the BOTDA interrogator. For instance, the Distributed Strain and Temperature Sensors (DSTS) BOTDA Interrogator configuration for the project was set-up as $5 \mathrm{~ns}$ pulse width corresponds to $50 \mathrm{~cm}$ in the fibre and sampling interval at every $5 \mathrm{~cm}$.

As for the project, a baseline reading configuration of Brillouin spectrum is determined as it will be used to measure the attenuation data along the cable with a certain spatial resolution. The commercial demodulation instrument; OZ Optics' Foresight ${ }^{\mathrm{TM}}$ series of fiber optic Distributed Strain andTemperature Sensors (DSTS) BOTDA module was able to reach a spatial resolution of $1 \mathrm{~ns}$, spatial step of $5 \mathrm{~cm}$, measuring accuracy of \pm 2 micron, strain range cover $-3 \%$ (compression) to $+4 \%$ (elongation) and maximum measurement is $100 \mathrm{~km}$.

\subsection{Construction of Soil Slope Model}

As illustrated in Figure 4, a small model test of finite soil slope was constructed in a steel chamber of $1.0 \mathrm{~m}$ length, $0.3 \mathrm{~m}$ width and $0.8 \mathrm{~m}$ height. An artificial slope model was built 
in the chamber with slope height and crest length of $0.3 \mathrm{~m}, 27$ degree of slope inclination and base thickness of $0.2 \mathrm{~m}$. Residual soil Grade VI has been used for the model material and has been obtained at one of Universiti Teknologi Malaysia premises ; P16 block of Electrical Engineering faculty. The soil was a mixture of $31 \%$ sand, $40 \%$ silt and $29 \%$ clay which categorized as fine-grained material. The residual soil is named as sandy SILT based on British Soil classification system. The slope model has been constructed by filling the chamber with the soil and subsequently proceeded by compaction. The compaction was done by tamping the soil layer by layer at a thickness of $100 \mathrm{~mm}$ each. The soil-water mass for each layer was pre-determined from a soil-phase back-calculation of targeted density. The targeted density was acquired from a correlation of suction, as well as volumetric and gravimetric moisture content. A rainfall simulator was fabricated from a 10-inch Polyvinyl chloride (PVC) pipe and the nozzles were designed at 8-cm center to center. The water supply of rainfall simulator was connected to the chamber through pumping system and was manually calibrated to acquire designated local rainfall intensity.

\subsection{Arrangement of Sensing Cable}

Figure 5 shows the arrangement of soil-embedded optical fibre for the laboratory experiments. As the optical fibre would be directly embedded in to the soil mass, fibre has to be proctected from a damage due to extrinsic factor such as poor splicing or bitten by the rodent. The 12-ribbon Fujikura optical fibre was used as the sensing cable because of the design of outer cladding has provided a better surface area compared to other single core optical fibre with circular cross-section which had assisted the strain transfer mechanism to the optical fibre core (refer Figure 3). The optical fibre cable were placed in horizontal direction perpendicular to surcharge load position. The three layers of sensing cables were laid at depths of $0.1 \mathrm{~m}, 0.2 \mathrm{~m}$ and $0.3 \mathrm{~m}$ from crest and represented as L3, L2 and L1 resepectively. A 1-meter-long unstrained fibre cables were located outside of the embedded sensing cables. The reason of doing so was because of the inherent property of Brillouin-based sensors were able to measure changes for both strain and temperature. Thus, the free-strained cable was to eliminate the temperature effect to the readings of strain. Besides, this isolation cable would also benefited as to define boundary to every layer and would be very helpful for a lengthy and wider model sample .
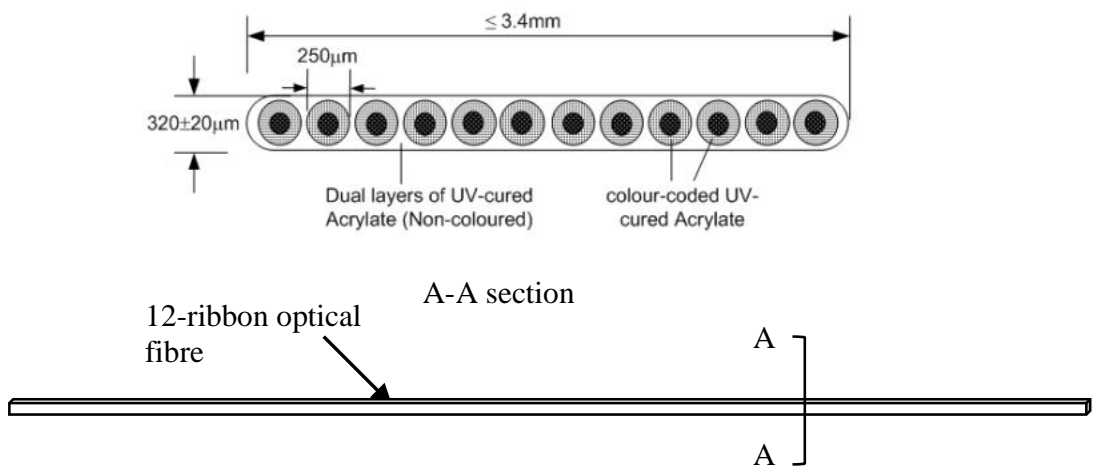

Fig. 3. A schematic diagram of sensing fibre [12](modified from Mohamad 2008). 


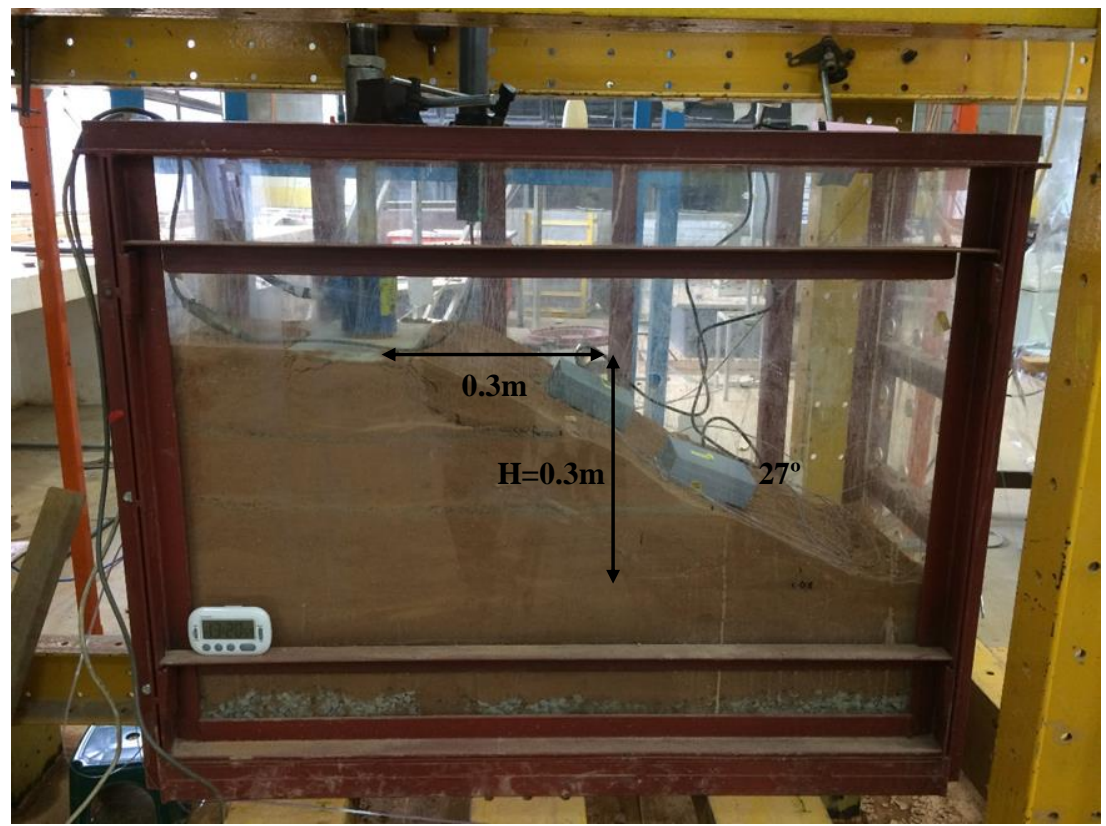

Fig. 4. An example of 27 degree soil slope model. 

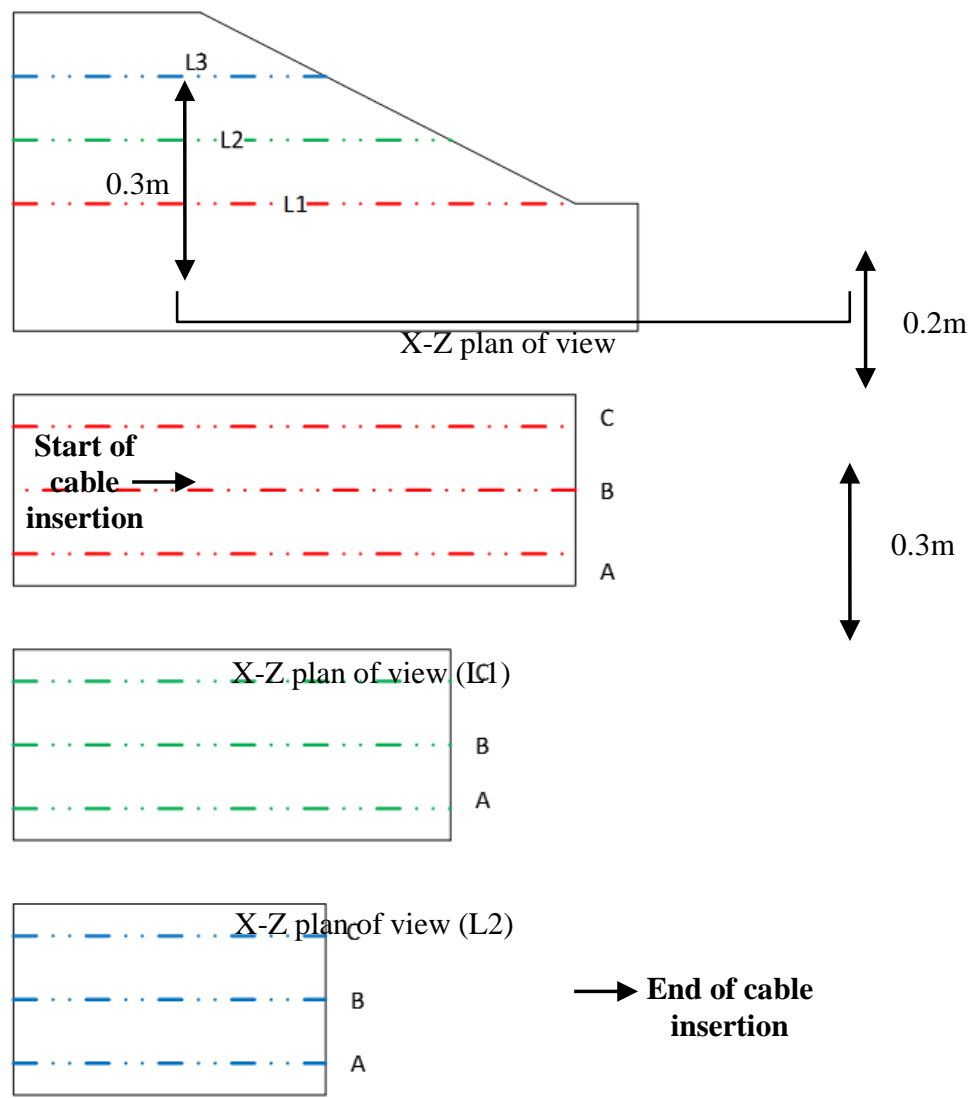

X-Z plan of view (L3)

Fig. 5. A two-dimensional orthographic projection of 27 degree inclination of finite slope.

\section{BOTDA Strain Results}

Figure 6 shows the strain distribution along the cable subjected to surcharge load. The positive microstrains curve signify the tensile reaction of optical fibre and the negative microstrains resembles compression strains in fibre[13]

From the graph, Layer 1 (L1) is representative of the lowest elevation of optical fibre layering, Layer 2 (L2) is positioned in the middle height of slope and Layer 3 (L3) is located adjacent to the slope crest. Based on Figure 6, the maximum microstrains were observed at L3 when the surcharge loading was at lower value $(2 \mathrm{kN}$ and $4 \mathrm{kN})$. This was reasonable to the stress distribution concept, whereby higher strain would occur at the nearest position to the loading plate. As for the subsequent loading of $7 \mathrm{kN}$ and $9 \mathrm{kN}$, the incremental microstrains were seen to maximised at position of L2 and optical fibre at position L3 was seen not responsive to the seqeuential loadings. The incremental microstrains were slightly increased when load at $9 \mathrm{kN}$ for both layer 2 (L2) and layer 1 (L1) and a downward pattern of strain curve was perceived at layer 3 (L3). After the load was increased to $10 \mathrm{kN}$, the strain measurements suddenly dropped for all layers and at position 2, there was an abrupt value of negative strain has been acquired in the distribution. Nonetheless, the mobilisations of fibre microstrains were not identical as stated by Wang et al 2009 [14] as the strain were seen to attenuate from top to bottom of slope 
model but no similar form for the present case study. In addition, the downward negative curve of strain profile at position of L3 (position 1) illustrates the optical fibre had utterly not responding to the deformation of soil mass. The negative values are characterized as free-state behaviour of optical fibre in the soil mass (Song et al 2016). The state where the optical fibre were no longer effective as the strain-sensing cable because of already not codeformed with the soil mass. This phenomena was also supported by Zeng et al. 2002[15] whereby optical fibre could not responsive to compression.

Figure 7 shows the slope failure feature for $1 \mathrm{~g}$ laboratory model at $9 \mathrm{kN}$ of surcharge load (total load surcharge was equal to $10 \mathrm{kN}$ ). During the laboratory test, the shear band has been visible at position 1 after a $9 \mathrm{kN}$ load. When the proceeded load of $10 \mathrm{kN}$ was induced on the load plate, the shear band was continuously to develop deeper towards the second layer (L2) of slope model but was not completely obvious by naked eyes. The shear plane was expected to advancing from position 1 to 2 when the loads were constantly increased because of external load on the crest would reduce slope stability. The surcharge load was set-up as a stress-controlled loading from the phneumatic cylinder and from the observation, the deformation feature illustrated the soil has failed due to undrained loading behaviour (refer to number 4 in Figure 7, the failure develops in horizontal). Therefore, the maximum tensile microstrain has formed near to the wall chamber which founded at L2B fibre cable (refer Figure 6) because of the reaction between optical fibre and deformed soil. However, the tensile microstrains were not replicated the same pattern at the other edge (slope face) because of the soil particles had disintegrated with the fibre.

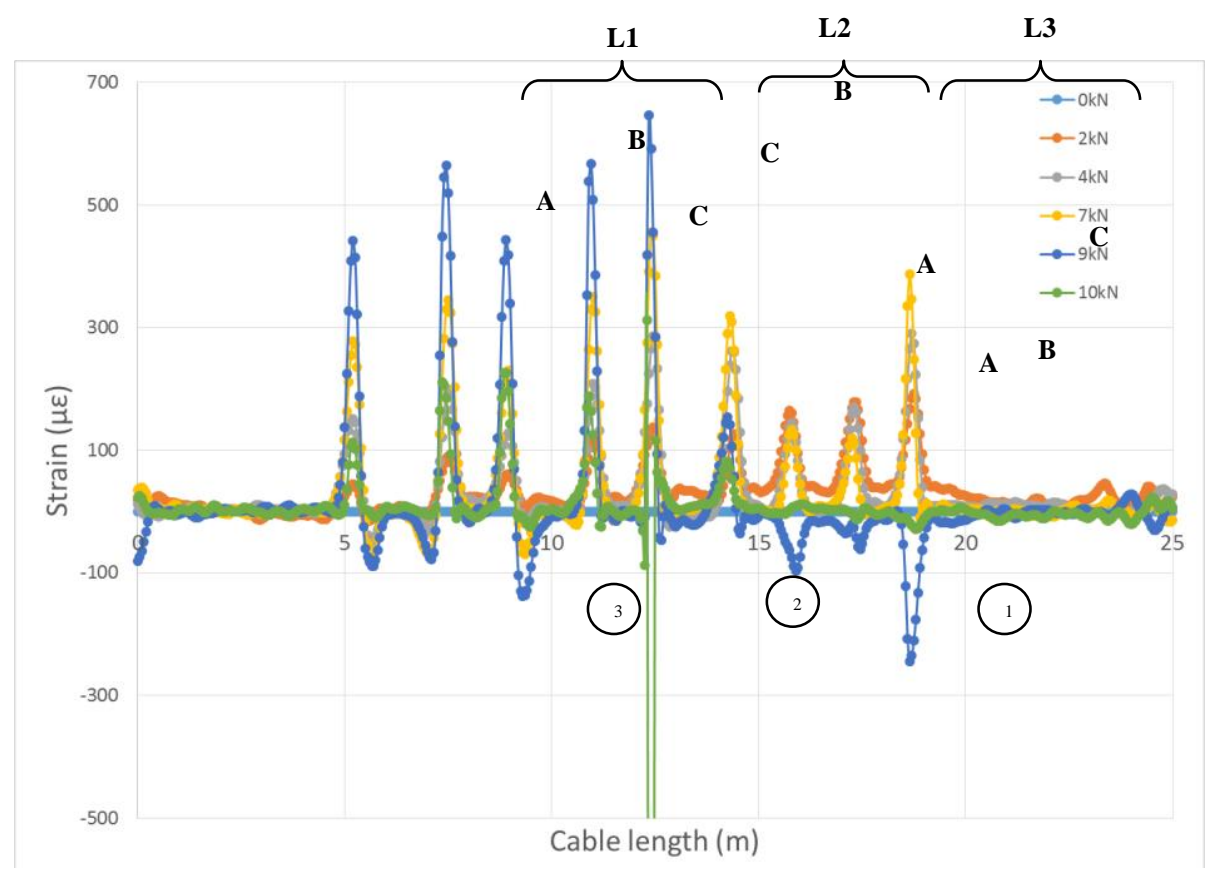

Fig. 6. Strain distribution of soil slope (slope inclination 27 degree). 


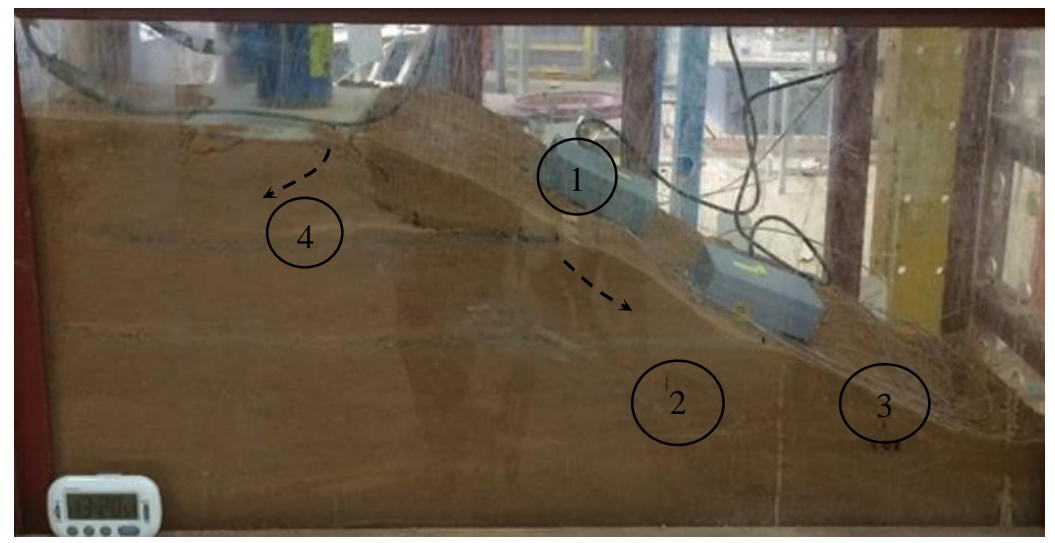

Fig. 7. Slope failure feature of a $1 \mathrm{~g}$ model (at $9 \mathrm{kN}$ of surcharge load).
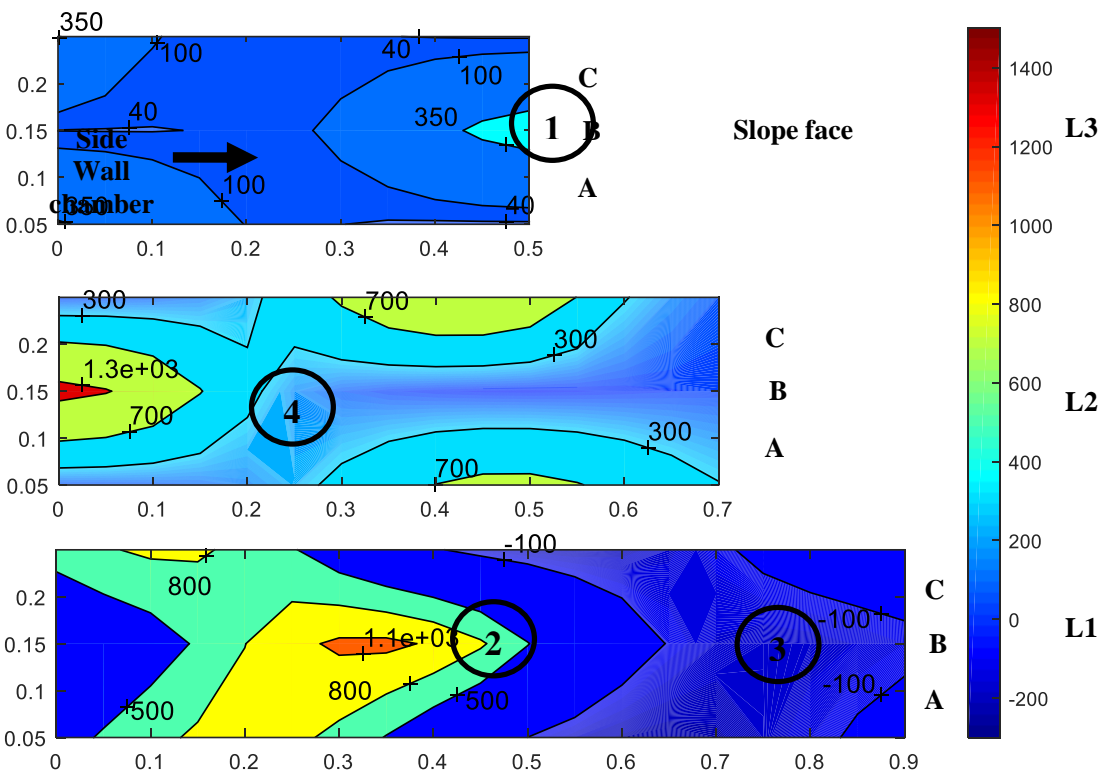

Fig. 8. Fibre cumulative strain contour at $9 \mathrm{kN}$ of surcharge load.

Figure 8 displays the cumulative fibre strain countour at $9 \mathrm{kN}$ of loading; the lowest microstrains were denoted in blue colour and red colour indicated the highest values of horizontal fibre strains. Layer 1 has actually illustrated a significant pattern of fibre strain distribution by which the horizontal strains had accumulated at the central region of slope and had reduced towards the slope face. The strains contour has signified the development of fibre cable strains in the soil mass which also indicated the condition of soil strain state during the loading. Indeed, the strain contour is more evident to show the development of soil strain within the soil mass and formation of a slip surface. Nonetheless, progression of fibre strains in this research study were not directly clarified the potential slip surface location. The developed tensile strains were more likely a resemblance to how much the optical fibre resisted or responded to soil particle re-arrangement. Moreover, inconsistency of position of peak strains in the model slope also has proven that a proper layout 
configuration is important in order to capture a realistic shear band advancement in a soil mass.

A verification of the above-mentioned verdicts were analysed via numerial modelling using the established Geostudio 2007 software. In this study, SLOPE/W, SEEP/W and SIGMA/W were used to simulate the laboratory model condition. An initial condition was established in SEEP/W finite element software and factor of safety, $F_{s}$ was later attained in the SLOPE/W limit equilbrium in order to observe the slope stability condition of locally loaded slopes. theMorgenstern and Price analysis method was opted to locate the optimum shear plane and subsequently calculate the minimum factor of safety $F_{s}$. The Mohr-Coulomb material model was selected to simulate the $1 \mathrm{~g}$ model in a plane strain condition. The soil properties as in material key-in step in SLOPE/W were as followed; unit weight $16 \mathrm{kN} / \mathrm{m}^{3}$, cohesion $8 \mathrm{kPa}$ and phi, $\varphi$ equal to 29 degree. These values were acquired from laboratory strength property test. The increased of loading has caused a reduction of safety factor and the slope has yet to fail (failure considers $F_{s}$ is less than 1). The factor of safety was gradually reduced from 3.422 (surcharge load equals to $2 \mathrm{kN}$ ) to 1.269 which at the maximum loading. Yet, the safety factor of a slope could neither explain the stress state nor strain state in order to ascertain the association of strain mobilisation and slope stability. This is due to the restriction of SLOPE/w software which only worked based on limit equilibrium method. Hence, SIGMA/W would be used to evaluate the stress state; both soil and pore pressure stress and also the strain behaviour of the slope model.

\section{Conclusions}

The accumulation of horizontal strains as illustrated in Figure 8 has proven that soil deformation were detected by the optical fibre sensor. The maximum incremental strain was approximately about 700 micro strains and the optical fibre were seized-up to codeformed with the soil mass at the most upper layer (L3) of strain-sensing cable layout started from $7 \mathrm{kN}$ of imposed load. At $9 \mathrm{kN}$ of loading, failure plane features of slope has been obviously seen and loading was then increased by $1 \mathrm{kN}$ which resulted a drastic decrement of strains reading. From SLOPE/W analysis, the factor of safety, $F_{s}$ obtained for the sequential surcharge loading on slope was reduced from 3.422 to 1.269. The numerical simulation shows the potential slip surface and it was found to be almost similar behaviour to that laboratory findings. In this paper, it can be concluded that BOTDA sensing system was able to function as an alternative sensor to measure soil deformation rather than using conventional strain gauges which usually were difficult to handle during instrumentation procedure. Moreover, the new technique of monitoring using optical fibre would promise a better results due to its 'distributed' nature of collecting data. Because of the uniqueness of its nature, optical fibre was successfully integrated with existing displacement monitoring method; for instance inclinometer in order to enhance the quality of slope monitoring data. It can be assured that the new technique of monitoring using optical fibre would be known in Malaysia for future geo-structures health monitoring programme Therefore, it is important to understand the state-of-art of optical fibre and the limitation of optical fibre equipment (BOTDR or BOTDA sensing system) in order to acquire the most representative monitoring data of soil movement.

The corresponding author would line to gratefully acknowledge the financial support provided by Research University Grant (RUG) of UTM; Tier 1 Vote 11H04 which had made the research project workable. 


\section{References}

1. C. K. Y. Leung et al., "Review: optical fiber sensors for civil engineering applications," Mater. Struct., pp. 871-906, 2013.

2. K. O. Hill, Y. Fujii, D. C. Johnson, and B. S. Kawasaki, "Photosensitivity in optical fiber waveguides: Application to reflection filter fabrication," Appl. Phys. Lett., vol. 32, no. 10, pp. 647-649, 1978.

3. H. H. Zhu, B. Shi, J. F. Yan, J. Zhang, and J. Wang, "Investigation of the evolutionary process of a reinforced model slope using a fiber-optic monitoring network," Eng. Geol., vol. 186, pp. 34-43, 2015.

4. A. Hartoq, An Introduction to Distributed Optical Fibre Sensors. CRC Press Taylor \& Francis Group, 2017.

5. T. Horiguchi and M. Tateda, "BOTDA-nondestructive measurement of single-mode optical fiber attenuation characteristics using Brillouin interaction: theory," J. Light. Technol., vol. 7, no. 8, pp. 1170-1176, 1989.

6. J. Liu, Y. Wang, Y. Lu, J. Wei, and D. P. Kanungo, "Application of distributed optical fiber sensing technique in monitoring the ground deformation," J. Sensors, vol. 2017, 2017.

7. A. Barrias, J. Casas, and S. Villalba, "A Review of Distributed Optical Fiber Sensors for Civil Engineering Applications," Sensors, vol. 16, no. 5, p. 748, 2016.

8. L. Zeni et al., "Brillouin optical time-domain analysis for geotechnical monitoring," J. Rock Mech. Geotech. Eng., vol. 7, pp. 1-5, 2015.

9. B. Madjdabadi, B. Valley, M. B. Dusseault, and P. K. Kaiser, "Experimental evaluation of a distributed Brillouin sensing system for measuring extensional and shear deformation in rock," Meas. J. Int. Meas. Confed., vol. 77, pp. 54-66, 2016.

10. J. fan Yan, B. Shi, F. Ansari, H. hu Zhu, Z. pu Song, and E. Nazarian, "Analysis of the strain process of soil slope model during infiltration using BOTDA," Bull. Eng. Geol. Environ., vol. 76, no. 3, pp. 947-959, 2017.

11. P. R. Orrell and A. P. Leach, "Fiber Optic Distributed Temperature Sensing," Proc. Soc. Photo-Optical Instrum. Eng., vol. 1777, p. 154, 1992.

12. H. Mohamad, "Distributed Optical Fibre Strain Sensing of Geotechnical Structures," Cambridge University, 2008.

13. H.-H. Zhu, Z. Wang, B. Shi, and J. K. Wong, "Feasibility study of strain based stability evaluation of locally loaded slopes: Insights from physical and numerical modeling," Eng. Geol., 2016.

14. B.-J. Wang, K. Li, B. Shi, and G. Wei, "Test on application of distributed fiber optic sensing technique into soil slope monitoring," Landslides, vol. 6, no. August 2008, pp. 61-68, 2009.

15. X. Zeng et al., "Strain measurement in a concrete beam by use of the Brillouin-scatteringbased distributed fiber sensor with single-mode fibers embedded in glass fiber reinforced polymer rods and bonded to steel reinforcing bars.," Appl. Opt., vol. 41, no. 24, pp. 5105$5114,2002$. 